\title{
Identification of potential COVID-19 main protease inhibitors using structure-based pharmacophore approach, molecular docking and repurposing studies
}

SAFA DAOUD ${ }^{1, *}$

SHADA J. ALABED ${ }^{2}$

LINA A. DAHABIYEH ${ }^{2}$

${ }^{1}$ Department of Pharmaceutical Chemistry and Pharmacognosy Faculty of Pharmacy, Applied Science Private University, Amman, Jordan

${ }^{2}$ Department of Pharmaceutical Sciences, School of Pharmacy

The University of Jordan Amman, Jordan
Accepted June 2, 2020

Published online June 3, 2020

\begin{abstract}
The current outbreak of novel coronavirus (COVID-19) infections urges the need to identify potential therapeutic agents. Therefore, the repurposing of FDA-approved drugs against today's diseases involves the use of de-risked compounds with potentially lower costs and shorter development timelines. In this study, the recently resolved X-ray crystallographic structure of COVID-19 main protease (Mpro) was used to generate a pharmacophore model and to conduct a docking study to capture antiviral drugs as new promising COVID-19 main protease inhibitors. The developed pharmacophore successfully captured five FDA-approved antiviral drugs (lopinavir, remdesivir, ritonavir, saquinavir and raltegravir). The five drugs were successfully docked into the binding site of COVID-19 Mpro and showed several specific binding interactions that were comparable to those tying the co-crystallized inhibitor X77 inside the binding site of COVID-19 Mpro. Three of the captured drugs namely, remdesivir, lopinavir and ritonavir, were reported to have promising results in COVID-19 treatment and therefore increases the confidence in our results. Our findings suggest an additional possible mechanism of action for remdesivir as an antiviral drug inhibiting COVID-19 Mpro. Additionally, a combination of structure-based pharmacophore modeling with a docking study is expected to facilitate the discovery of novel COVID-19 Mpro inhibitors.
\end{abstract}

Keywords: COVID-19, main protease, pharmacophore, structure-based modeling, docking study, remdesivir, repurposing

The World Health Organization (WHO) was informed on December 2019 about a cluster of unexplained severe pneumonia cases in Wuhan, China, known later to be caused by a novel beta coronavirus (1). Coronavirus is an enveloped, positive-sense and single-stranded RNA virus from the genus Betacoronavirus that is distributed in birds, humans and other mammals $(2,3)$. WHO has named this novel coronavirus disease as COVID-19 (4). A novel coronavirus has resulted in an ongoing outbreak of viral pneumonia all over the world, forcing the WHO to declare that the outbreak of this deadly and fast-spreading

\footnotetext{
*Correspondence; e-mail: s_daoud@asu.edu.jo
} 
infectious pneumonia represents a global health emergency (5). Despite rigorous global containment and quarantine efforts, the incidence of COVID-19 continues to rise. According to WHO official records, as of May $31^{\text {st }}, 2020$, there have been 5,934,936 infected people globally and 367,166 confirmed deaths in 216 countries, areas or territories with reported cases of the disease (6).

At present, there are no specific effective antiviral drugs or vaccines against COVID-19 infection. Current treatments are mainly directed towards supporting the symptomatic and respiratory conditions. Therefore, there is an urgent need to new studies and approaches that facilitate the identification of effective therapy that fight such life-threatening virus (7). Under the current global COVID-19 pandemic, drug repurposing represents an attractive and promising approach to identify possible treatment strategies using already FDA-approved marketed drugs (8). Obviously, the repurposing of such drugs against today's diseases involves the use of de-risked compounds with potentially lower overall costs and shorter development timelines $(9,10)$. Computer-aided drug design techniques represent a very efficient approach to provide promising results in drug discovery. Structure-based pharmacophore approach has proven to be useful for supporting in silico hit discovery, hit-to-lead expansion, and lead optimization $(11,12)$. The protein-ligand complex based approach is specialized to gain insights into ligand-protein interactions and to identify the essential pharmacophore features necessary for optimal interaction and biological activity (13). Furthermore, structure-based pharmacophore models obtained using single ligand-target structures have proven their worth as an alternative to the traditional approaches. This is particularly evident by their role in the virtual screening process, where they have taken center stage in many studies $(11,14)$.

The COVID-19 virus encodes two polyproteins that are crucial for viral replication and transcription; ppla and pplab. COVID-19 virus' main protease (Mpro), also referred to as 3-chymotrypsin-like protease (3C-like protease), plays a pivotal role in releasing the functional polypeptides from the polyproteins (15). Therefore, Mpro represents a new attractive drug target for the discovery of potential therapeutics against COVID-19.

In this study, we aim to apply a structure-based pharmacophore drug design approach combined with a docking study as a quick approach to identify promising FDA-approved antiviral drugs that can target COVID-19 virus main protease.

\section{METHODS}

\section{Pharmacophore generation}

Pharmacophore generation and docking studies were performed using DiscoveryStudio 2.5.5 from Accelrys Software (BIOVIA, USA). The 3D coordinates of COVID-19 Mpro complexed with inhibitor, X77, were downloaded from the Protein Data Bank (PDB code: 6W63, $2.10 \AA$ ) (https://www.rcsb.org/). Hydrogen atoms were added to the proteins utilizing DiscoveryStudio 2.5.5 templates for protein residues and the water molecules were kept in the protein structure. The protein-inhibitor complex was carefully explored to identify the intermolecular interactions; only those interactions located within a maximum of $3 \AA$ distance were considered. A structure-based pharmacophore approach that relies on these interactions was conducted and manual pharmacophore generation was carried on. 


\section{Screening FDA-approved antiviral drugs}

Based on a literature survey, 36 FDA-approved antiviral drugs were used in this study including abacavir, acyclovir, amprenavir, asunaprevir, atazanavir, boceprevir, daclatasvir, danoprevir, darunavir, dolutegravir, efavirenz, entecavir, etravirine, favipiravir, fosamprenavir, galidesivir, ganciclovir, grazoprevir, indinavir, lamivudine, lomibuvir, lopinavir, nelfinavir, nevirapine, penciclovir, raltegravir, remdesivir, ribavirin, rilpivirine, ritonavir, saquinavir, simeprevir, telaprevir, tipranavir, triflurdine and umifenovir. The 3D structure of each drug was downloaded from the PubChem database (https://pubchem. ncbi.nlm.nih.gov/) and a list in (SD) format was prepared.

The prepared list was mapped against our developed pharmacophore employing the "Caeser-Flexible ligand pharmacophore mapping" option implemented within CATALYST (HYPOGEN module), as an attempt to identify potential COVID-19 protease inhibitors.

\section{Docking study}

COVID-19 Mpro crystal structure (PDB code: 6W63, 2.10 ̊̊) was utilized in subsequent docking experiments without energy minimization. Explicit water molecules were kept in the binding pocket. The docking experiment was adapted from the team's previous publications $(16,17)$ using the site-feature docking algorithm (LibDock). A sphere of $7.64 \AA$ radius based on the co-crystallized ligand was used to define the binding site. LibDock is a docking algorithm that positions the generated ligand conformations in the protein active site based on polar and apolar interaction sites (hotspots). It uses standard Catalyst engine in DiscoveryStudio 2.5.5 to generate conformations using "best" option (a maximum of 250 conformers in which each of them not exceeding an energy threshold of $20 \mathrm{kcal} \mathrm{mol}^{-1}$ from the most stable conformer) with final CHARMm minimization step to optimize the docked poses $(18,19)$.

Top poses were kept for subsequent scoring where 7 docking-scoring functions were employed [Jain (20), LigScore1, LigScore2 (21, 22), PLP1, PLP2 (23), PMF and PMF04 (24, 25)]. The docking and scoring settings that had closely reproduced the crystallographic pose of the potent inhibitor X77 were selected to carry docking experiments for antiviral drugs that were captured by the generated pharmacophore.

\section{RESULTS AND DISCUSSION}

The urgent need for treatments during the COVID-19 pandemic makes computeraided drug design a very efficient technique to apply, in order to quickly identify promising drug repurposing candidates, especially after the detailed 3D-structures of various key proteins are resolved $(26,27)$. In this study, COVID-19 Mpro, or 3CL-protease, was used to generate a pharmacophore model and to perform a docking experiment to identify potential COVID-19 Mpro inhibitors from the FDA-approved antivirals, and hence, facilitate the repurposing of these drugs as potential treatments for COVID-19. The COVID-19 Mpro is the main protease used to cleave polyproteins into replication-related proteins and, thus, plays a crucial role in mediating viral replication and transcription (15). The 3CL-protease structure (PDB code: 6W63) was obtained from the RCSB Protein Data Bank which was recently released on March 25th, 2020 (28). It goes without saying that the information 


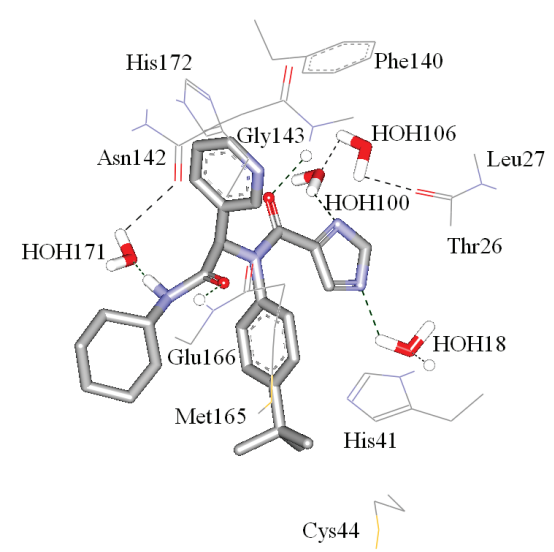

Fig. 1. Detailed view of interactions of different amino acid residues within the binding pocket of COVID-19 Mpro with the potent co-crystallized inhibitor X77 (PDB code: 6W63). Hydrogen bonding interactions are shown as dotted black lines.

Table I. Coordinates of the developed COVID-19 main protease pharmacophore

\begin{tabular}{ccccccccc}
\hline & HBA & HBA & HBA & HBA & HBD & RingArom & RingArom & Hbic \\
\hline X & -21.494 & -19.343 & -19.228 & -16.85 & -22.267 & -16.883 & -20.274 & -21.498 \\
Y & 17.268 & 24.35 & 25.136 & 16.203 & 21.457 & 20.223 & 16.561 & 14.523 \\
$Z$ & -34.948 & -32.339 & -29.25 & -25.921 & -24.868 & -24.4 & -26.164 & -29.359 \\
\hline
\end{tabular}

HBA - hydrogen bond acceptor, HBD - hydrogen bond donor, RingArom - aromatic rings, Hbic - hydrophobic feature

about the protein structure from its X-ray crystal structures is a good source to generate the structure-based pharmacophore (14).

Initially, the X-ray crystallographic structure of Mpro complexed with the inhibitor X77 was carefully inspected to identify the protein-ligand interactions. Fig. 1 shows the binding interactions anchoring the potent inhibitor X77 within COVID-19 Mpro binding site. Apparently, the co-crystalized inhibitor binds into the binding site via several hydrogen bonding, $\pi$-stacking and hydrophobic interactions. The potent inhibitor X77 is involved in five hydrogen-bonding interactions connecting its amidic $\mathrm{NH}$ with the carbonyl of Asn142 via bridging water molecule, the amidic carbonyl with $\mathrm{NH}$ of Glu166, the other carbonyl with $\mathrm{NH}$ of Gly143, imidazole N with Thr26 via two bridging water molecules, and the fifth hydrogen bond interaction connects the second imidazole $\mathrm{N}$ with $\mathrm{NH}$ of His41. Additionally, two $\pi-\pi$ stacking interactions are noted. First, between the benzene ring of the potent inhibitor X77 and the imidazole ring of His41, where the second $\pi-\pi$ stacking involves inhibitor's pyridine ring where it sandwiched between the benzene ring of Phe140 and the imidazole ring of His172. Furthermore, the projected tert-butyl of X77 


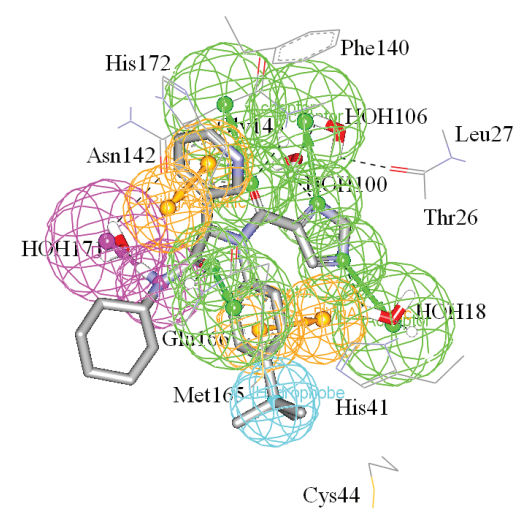

Fig. 2. Manual generation of the pharmacophore as guided by the interactions of the potent inhibitor within the binding pocket of COVID-19 main protease (Fig. 1).

towards Met165 and Cys44 suggests the existence of mutual hydrophobic interactions that anchor this inhibitor within the binding pocket of COVID-19 Mpro.

The former interactions between the potent inhibitor X77 and the binding pocket of COVID-19 Mpro were used to guide the manual generation of pharmacophore theory, where each interaction is translated into an equivalent feature (Fig. 2). The constructed pharmacophore theory includes eight features: four hydrogen bond acceptors (HBA), one hydrogen bond donor (HBD), two aromatic rings (RingArom) and one hydrophobic feature (Hbic) as shown in Fig. 3. XYZ coordinates for each feature are represented in Table I. It is well known that the selectivity of the pharmacophore is highly dependent on the number of features;

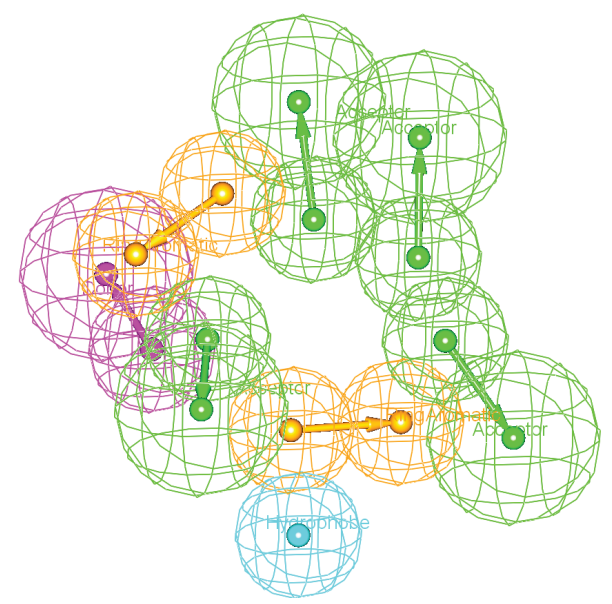

Fig. 3. The generated pharmacophore theory. HBA is shown as vectored green spheres, HBD as vectored pink spheres, Hbic is shown as a blue sphere and RingArom as vectored orange spheres. 

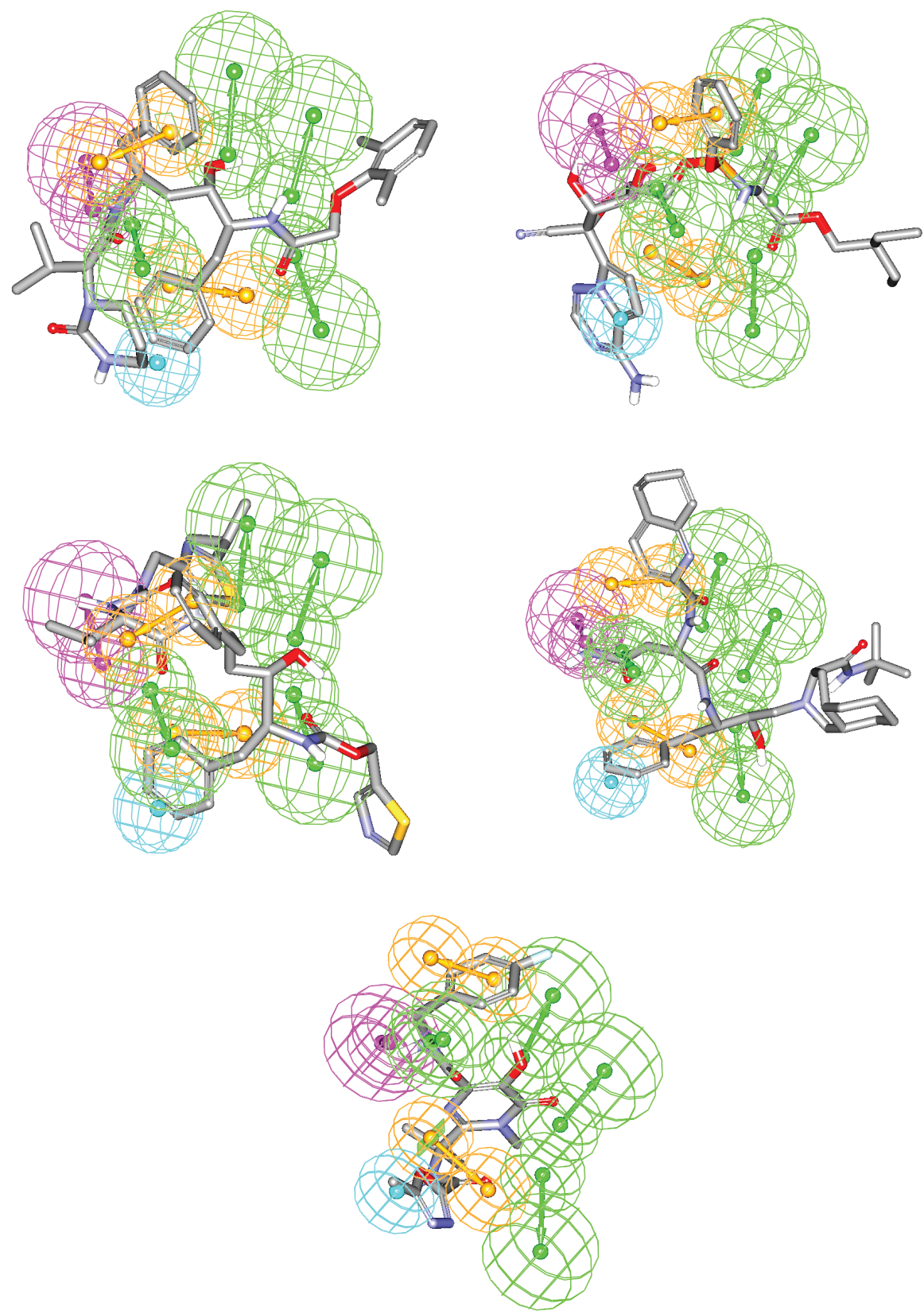

Fig. 4. Mapping of the five captured FDA approved drugs against the developed pharmacophore: a) lopinavir, b) remdesivir, c) ritonavir, d) saquinavir and e) raltegravir. 


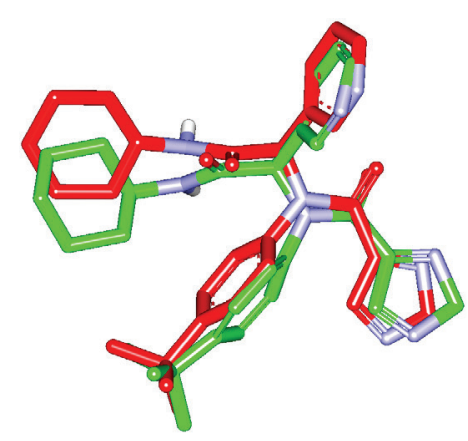

Fig. 5. Comparison between the docked pose of COVID-19 Mpro inhibitor X77 (green) as produced by docking simulation and the crystallographic structure of X77 within the COVID-19 Mpro binding pocket (red, PDB code: 6 W63), RMSD value $=0.93 \AA$.

complex pharmacophores with a high number of features are more selective than simple ones with a low number of features (29). The generated pharmacophore herein has eight features that will enhance its selectivity and, consequently, restrict the number of captured hits and increase the chance of correctly capturing true positive hits.

Based on the developed pharmacophore model, ligand pharmacophore mapping was performed using the prepared list of the 36 FDA-approved antiviral drugs to select potential drug-candidates. Out of the 36 drugs, our pharmacophore successfully captured five drugs namely: lopinavir, remdesivir, ritonavir, saquinavir and raltegravir (supplementary material, Fig. S1). Fig. 4 shows how these five drugs mapped against the developed pharmacophore.

To further increase the confidence of our results, a docking study for the five captured antiviral drugs into the catalytic site of COVID-19 Mpro was conducted. Before performing a docking study, the docking settings used herein were validated by comparing the crystallographic pose of the potent inhibitor X77 complexed within COVID-19 Mpro with the docked pose of the same ligand. The docking settings and the scoring function Ligscore 2 closely reproduced the crystallographic pose of X77 (Fig. 5) with root mean square difference (RMSD) value of $0.93 \AA$ A providing confidence to dock the five captured antiviral drugs using the same settings. Interestingly, the five captured antivirals were successfully docked inside the binding pockets of COVID-19 Mpro. Fig. 6 shows the binding interactions tying these antiviral drugs within the Mpro binding pocket as proposed by the docking study. It appears that the antiviral drugs can be involved in several binding interactions within the binding pockets of COVID-19 Mpro (hydrogen bonding, hydrophobic and stacking attractions) comparable to the binding interactions tying co-crystallized ligands X77 into this protease, as stated earlier.

The docked pose of lopinavir is involved in four hydrogen bondings with $\mathrm{NH}$ of Glu166, carbonyl of Leu167 and Asn142 via bridging water molecule and the carboxylate of Glu166 via water molecule also. Additionally, $\pi$-stacking interactions within the binding pocket of Mpro were noticed between one of the benzene rings of lopinavir against the amide of Gln189 indicating mutual $\pi$-amide stacking interaction. The other benzene ring is docked at close proximity to the aromatic side chain of Phe140 while dimethylbenzene is sandwiched between Tyr54 and His41 suggesting two mutual $\pi-\pi$ stackings (Fig. 6a). 

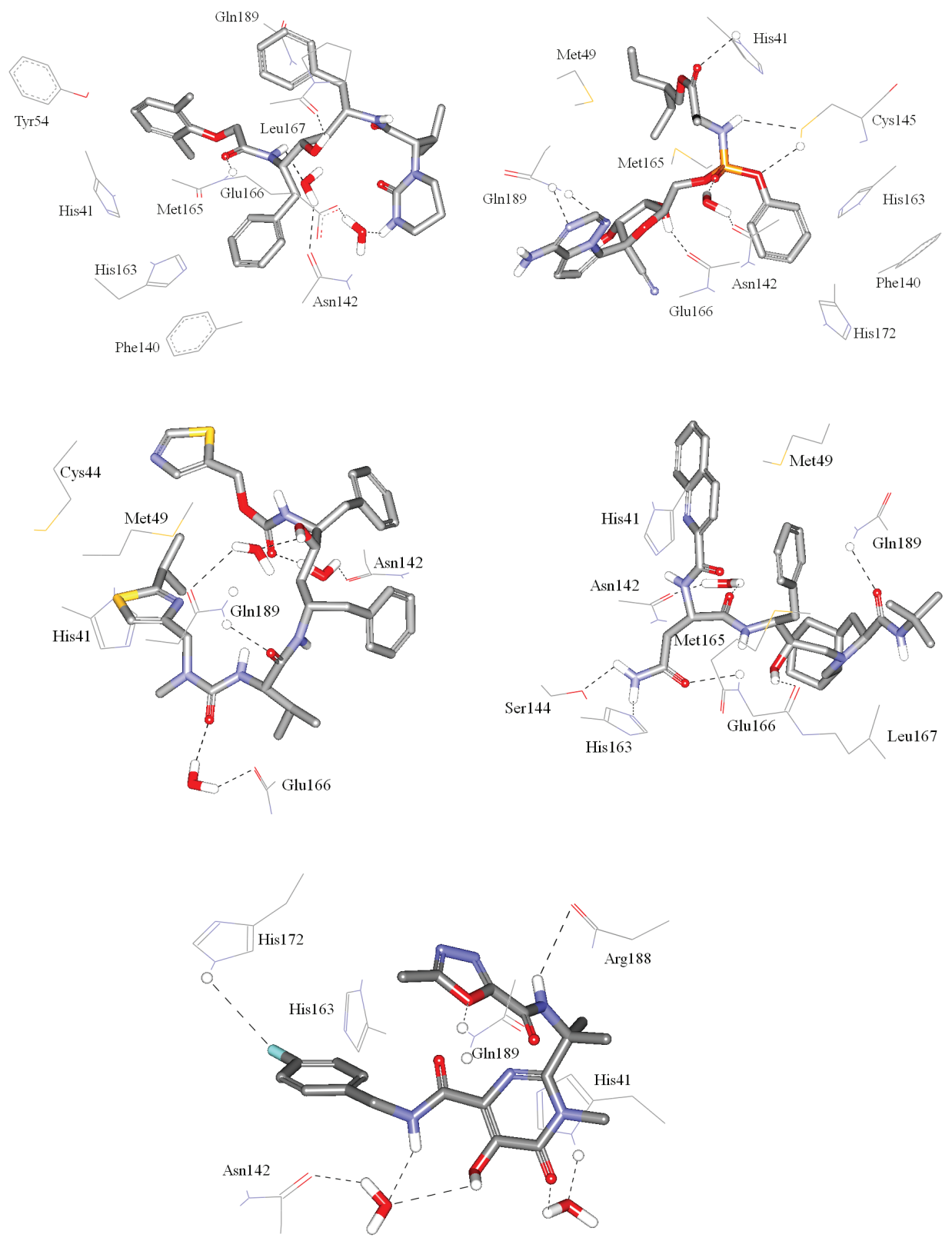

Fig. 6. Detailed view showing the interactions of different amino acid residues within the binding pocket of COVID-19 main protease (Mpro) with the docked antiviral drugs: a) lopinavir, b) remdesivir, c) ritonavir, d) saquinavir and e) raltegravir. Hydrogen bonding interactions are shown as dotted black lines. 
The docked pose of remdesivir is involved in seven hydrogen bondings with $\mathrm{NH}$ of His41, carbonyl of Asn142 via water molecule, carbonyl of Glu166, amidic $\mathrm{NH}_{2}$ of Gln189 and $\mathrm{SH}$ of Cys145. Moreover, the benzene ring of remdesivir is docked in close vicinity of the aromatic side chains of His163, Phe140 and His172, suggesting mutual $\pi-\pi$ stacking, where the lipophilic chain of remdesivir is very close to Met49 and Met165 side chains indicating the probability of hydrophobic interaction (Fig. 6b).

The docked pose of ritonavir is involved in four hydrogen bondings with amidic $\mathrm{NH}_{2}$ of Gln189, carbonyl of Glu166, amidic carbonyl of Gln189 and amidic carbonyl of Asn142, the last three interactions via bridging water molecules. Furthermore, ritonavir is involved in $\pi-\pi$ stacking with His41 and hydrophobic interaction with Cys44 and Met49 (Fig. 6c).

Likewise, the docked pose of saquinavir tying itself in Mpro binding pocket through six hydrogen bondings with the hydroxyl of Ser144, NH of His163, NH of Glu166, carbonyl of Glu166, amidic NH2 of Gln189 and the amidic carbonyl of Asn142 via a water molecule. Also, the close proximity to the aromatic ring of His41 and the side chain of Met49 suggesting the probability of $\pi-\pi$ stacking and hydrophobic interactions, resp. (Fig. 6d).

The docked pose of raltegravir is involved in hydrogen bonding interactions with the amidic $\mathrm{NH}_{2}$ of Gln189, the carbonyl of Arg188, NH of His172, NH of His41 and the carbonyl of Asn142, the latter two interactions via bridging water molecules. Similarly, the close proximity to aromatic side chains of His41, His163 and His172 suggesting $\pi-\pi$ stacking interactions (Fig. 6e).

The results obtained from the docking study support the outcomes of pharmacophore model mapping and confirm that the five antiviral drugs captured by the generated pharmacophore model could be involved in several binding interactions within the binding pockets of COVID-19 Mpro. Most importantly, these interactions are comparable to the binding interactions tying the co-crystallized inhibitor X77 into the Mpro binding site.

Interestingly, three of the captured drugs, lopinavir, remdesivir and ritonavir, have been clinically tested as a potential treatment for COVID-19 (30). Remdesivir is a nucleotide analog inhibitor of the Ebola virus RNA-dependent RNA polymerase (31) and has shown promising results in the treatment of COVID-19 infection (32). The ability of the developed pharmacophore herein to capture remdesivir (Fig. 4b) and the numerous binding interactions that anchor remdesivir within the binding site of COVID-19 Mpro (Fig. 6b) suggest that the potential ability of remdesvir to inhibit COVID-19 Mpro might contribute to its reported useful clinical effect in COVID-19 cases. Our results provide an additional possible mechanism of action for remdesivir as an antiviral drug. Needless to say, dual inhibitors are much more efficient and have more favorable outcomes (33). Lopinavir and ritonavir, HIV protease inhibitors approved for acquired immune deficiency syndrome (AIDS) treatment in several countries (34), were both captured by the pharmacophore (Figs. 4a,c) and successfully docked in Mpro binding sites (Figs. 6a,c). Although, currently there is no strong evidence for the efficacy of lopinavir/ritonavir in the treatment of COVID-19 (35), Cao et al. (36) have reported that hospitalized patients with severe COVID-19 who received lopinavir/ritonavir had slightly lower 28-day mortality rate. The agreement of our findings with the results from reported clinical trials proves the validity of the approach followed in this study. The remaining two captured antiviral drugs, saquinavir and raltegravir, showed several interactions within COVID-19 Mpro suggesting that these two drugs might act as promising COVID-19 main protease (Mpro) inhibitors. However, to the best of our knowledge, until now there are no clinical studies regarding the effectiveness of these two drugs as COVID-19 treatments. 


\section{CONCLUSIONS}

A simple structure-based approach was conducted to develop a pharmacophore model for COVID-19 main protease (Mpro) binding site. Five antiviral drugs were captured by the developed pharmacophore and docked inside the binding site of COVID-19 Mpro. The five antiviral drugs (lopinavir, remdesivir, ritonavir, saquinavir and raltegravir) showed specific binding interactions within Mpro binding pocket that were comparable to those tying the co-crystallized inhibitor X77. Our results agreed with clinical trials where three of the captured drugs, lopinavir, remdesivir and ritonavir, showed promising results in COVID-19 treatment. The remaining two drugs, saquinavir and raltegravir, are highly recommended to be taken into consideration while treating COVID-19. The strategy followed herein by combining structure-based pharmacophore modeling with a docking study is expected to facilitate the discovery of novel COVID-19 Mpro inhibitors by exploring different databases, including natural products or designing novel inhibitors based on the developed pharmacophore. In vitro bioassay using recombinant COVID-19 main protease was not conducted for the five captured hits due to time constraints with regards to shipment of the required materials. Additionally, only 36 FDA-approved antiviral drugs were used in this study. However, this is perceived to be acceptable as the main focus of the presented work was to provide evidence for the repurposing of the currently approved antivirals in the treatment of COVID-19. In the future, in vitro bioassay will be conducted for the five captured antiviral drugs to further confirm their biological activity, and in silico screening of several classes of drugs and bio- and agrochemicals will be conducted using the generated pharmacophore.

Abbreviations, acronyms, symbols. - AIDS - Acquired Immune Deficiency Syndrome, 3CL-protease -3C-like protease, CHARMm - Chemistry at Harvard Molecular Mechanics, COVID-19 - Corona Virus Disease of 2019, HBA - hydrogen bond acceptor, HBD - hydrogen bond donor, Hbic - hydrophobic, HIV - Human Immunodeficiency Virus, Mpro - main protease, PDB - Protein Data Bank, PLP - piecewise linear potential, PMF - potential mean force, RingArom - ring aromatic, RMSD - root mean square distance

Supplementary material available upon request.

\section{REFERENCES}

1. N. Zhu, D. Zhang, W. Wang, X. Li, B. Yang, J. Song, X. Zhao, B. Huang, W. Shi, R. Lu and P. Niu, A novel coronavirus from patients with pneumonia in China, 2019, N. Engl. J. Med. 382 (2020) 727-733; https://doi.org/10.1056/NEJMoa2001017

2. A. Zumla, J. F. Chan, E. I. Azhar, D. S. Hui and K. Y Yuen, Coronaviruses-drug discovery and therapeutic options, Nat. Rev. Drug Discov. 15 (2016) 327-347; https://doi.org/10.1038/nrd.2015.37

3. F. He, Y. Deng and W. Li, Coronavirus Disease 2019 (COVID-19): What we know? J. Med. Virol. (2020) (7 pages); https://doi.org/10.1002/jmv.25766

4. C. C. Lai, T. P. Shih, W. C. Ko, H. J. Tang and P. R. Hsueh, Severe acute respiratory syndrome coronavirus 2 (SARS-CoV-2) and coronavirus disease-2019 (COVID-19): The epidemic and the challenges, Int. J. Antimicrob. Agents 55 (2020) Article ID 105924; https://doi.org/10.1016/j.ijantimicag.2020.105924

5. J. She, J. Jiang, L. Ye, L. Hu, C. Bai and Y. Song, 2019 Novel coronavirus of pneumonia in Wuhan, China: emerging attack and management strategies, Clin.. Transl. Med. 9 (2020) Article ID 19; https://doi.org/10.1186/s40169-020-00271-z 
6. World Health Organization, Novel Coronavirus (Covid-19): Situation Report, 3. 2020, WHO, Geneve 2020; https://www.who.int/emergencies/diseases/novel-coronavirus-2019?gclid=CjwKCAjwq832B RA5EiwACvCWsSQ_-qAh4z8Z5_gvnayDI2PrSx4poydPpu-pvDZX-k34RQDqn8TgEBoChdkQAvD_BwE; last access date May 31, 2020

7. H. A. Rothan and S. N. Byrareddy, The epidemiology and pathogenesis of coronavirus disease (COVID-19) outbreak, J. Autoimmun. 109 (2020) Article ID 102433 (4 pages); https://doi.org/10.1016/j. jaut.2020.102433

8. S. L. Senanayake, Drug repurposing strategies for COVID-19, Future Drug Discov. 2 (2020) (3 pages); https://doi.org/10.4155/fdd-2020-0010

9. S. Pushpakom, F. Iorio, P. A. Eyers, K. J. Escott, S. Hopper, A. Wells, A. Doig, T. Guilliams, J. Latimer, C. McNamee and A. Norris, Drug repurposing: progress, challenges and recommendations, Nat. Rev. Drug Discov. 18 (2019) 41-58; https://doi.org/10.1038/nrd.2018.168

10. S. G. V. Rosa and W. C. Santos, Clinical trials on drug repositioning for COVID-19 treatment, Rev. Panam. Salud Públ. 44 (2020) e40; https://doi.org/10.26633/RPSP.2020.40

11. A. Gaurav, V. Gautam, S. Pereira, J. Alvarez-Leite, F. Vetri, M. Choudhury, D. Pelligrino, P. Sundivakkam, K. Radhakrishnan and A. Krieger, Structure-based three-dimensional pharmacophores as an alternative to traditional methodologies, J. Receptor Ligand Channel Res. 7 (2014) 27-38; https:// doi.org/10.2147/JRLCR.S46845

12. M. P. Sanders, R. McGuire, L. Roumen, I. J. de Esch, J. de Vlieg, J. P. Klomp and C. de Graaf, From the protein's perspective: the benefits and challenges of protein structure-based pharmacophore modeling, MedChem Comm. 3 (2012) 28-38; https://doi.org/10.1039/C1MD00210D

13. M. Wieder, U. Perricone, T. Seidel, S. Boresch and T. Langer, Comparing pharmacophore models derived from crystal structures and from molecular dynamics simulations, Monatsh. Chem. 147 (2016) 553-563, https://doi.org/10.1007/s00706-016-1674-1

14. M. Arooj, S. Sakkiah, S. Kim, V. Arulalapperumal and K. W. Lee, A combination of receptor-based pharmacophore modeling \& QM techniques for identification of human chymase inhibitors. PLoS One 8 (2013) e63030; https://doi.org/10.1371/journal.pone.0063030

15. Z. Jin, X. Du, Y. Xu, Y. Deng, M. Liu, Y. Zhao, B. Zhang, X. Li, L. Zhang, C. Peng, Y. Duan, J. Yu, L. Wang, K. Yang, F. Liu, R. Jiang, X. Yang, T. You, X. Liu, X. Yang, F. Bai, H. Liu, X. Liu, L. W. Guddat, W. Xu, G. Xiao, C. Qin, Z. Shi, H. Jiang, Z. Rao and H. Yang: Structure of Mpro from SARSCoV-2 and discovery of its inhibitors, Nature 5 (2020) (20 pages); https://doi.org/10.1038/s41586-0202223-y

16. L. A. Dahabiyeh, E. Y. Abu-rish and M. O. Taha, Inhibition of monoglyceride lipase by proton pump inhibitors: investigation using docking and in vitro experiments, Pharmacol. Rep. 72 (2019) 435-442 ; https://doi.org/10.1007/s43440-019-00013-0

17. L. A. Dahabiyeh, Y. Bustanji and M. O. Taha, The herbicide quinclorac as potent lipase inhibitor: Discovery via virtual screening and in vitro/in vivo validation, Chem. Biol. Drug Des. 93 (2019) 787-797; https://doi.org/10.1111/cbdd.13463

18. D. J. Diller and K. M. Merz, Jr, High throughput docking for library design and library prioritization, Proteins 43 (2001) 113-124; https://doi.org/10.1002/1097-0134(20010501)43:2<113::aidprot1023>3.0.co;2-t

19. S. N. Rao, M. S. Head, A. Kulkarni and J. M. LaLonde, Validation studies of the site-directed docking program LibDock, J. Chem. Inf. Model. 47 (2007) 2159-2171; https://doi.org/10.1021/ci6004299

20. A. N. Jain, Scoring noncovalent protein-ligand interactions: a continuous differentiable function tuned to compute binding affinities, J. Comput. Aided Mol. Des. 10 (1996) 427-440; https://doi. org/10.1007/BF00124474

21. A. Krammer, P. D. Kirchhoff, X. Jiang, C. Venkatachalam and M. Waldman, LigScore: a novel scoring function for predicting binding affinities, J. Mol. Graph. Model. 23 (2005) 395-407; https:// doi.org/10.1016/j.jmgm.2004.11.007 
22. C. M. Venkatachalam, X. Jiang, T. Oldfield and M. Waldman, LigandFit: a novel method for the shape-directed rapid docking of ligands to protein active sites, J. Mol. Graph. Model. 21 (2003) 289-307; https://doi.org/10.1016/s1093-3263(02)00164-x

23. D. K. Gehlhaar, D. Bouzida and P. A. Rejto, Reduced dimensionality in ligand-protein structure prediction: covalent inhibitors of serine proteases and design of site-directed combinatorial libraries, ACS Pub. 719 (1999); https://doi.org/10.1021/bk-1999-0719.ch019

24. I. Muegge, PMF scoring revisited, J. Med. Chem. 49 (2006) 5895-5902; https://doi.org/10.1021/ jm050038s

25. I. Muegge and Y. C. Martin, A general and fast scoring function for protein-ligand interactions: a simplified potential approach, J. Med. Chem. 42 (1999) 791-804; https://doi.org/10.1021/jm980536j

26. J. Wang, Fast identification of possible drug treatment of coronavirus disease-19 (COVID-19) through computational drug repurposing study, J. Chem. Inf. Model. (2020) (10 pages); https://doi. org/10.1021/acs.jcim.0c00179

27. A. Farag, P. Wang, M. Ahmed and H. Sadek, Identification of FDA approved drugs targeting COVID-19 virus by structure-based drug repositioning, ChemRxiv. (preprint) (2020); https://doi. org/10.26434/chemrxiv.12049647.v1

28. A. D. Mesecar (Center for Structural Genomics of Infectious Diseases, CSGID), A taxonomicallydriven approach to development of potent, broad-spectrum inhibitors of coronavirus main protease including SARS-CoV-2 (COVID-19), to be published; PDB ID 6W63, title: Structure of COVID-19 main protease bound to potent broad-spectrum non-covalent inhibitor X77, 2020; https://doi. org/10.2210/pdb6W63/pdb

29. J. Meslamani and D. Rognan, Protein-ligand pharmacophores: concept, design and applications, CICSJ Bull. 33 (2015) 27-32; https://doi.org/10.11546/cicsj.33.27

30. L. Dong, S. Hu and J. Gao, Discovering drugs to treat coronavirus disease 2019 (COVID-19), Drug Discov. Ther. 14 (2020) 58-60; https://doi.org/10.5582/ddt.2020.01012

31. E. P. Tchesnokov, J. Y. Feng, D. P. Porter and M. Götte, Mechanism of inhibition of Ebola virus RNA-dependent RNA polymerase by remdesivir, Viruses 11 (2019) Article ID 326; https://doi. org/10.3390/v11040326

32. J. A. Al-Tawfiq, A. H. Al-Homoud and Z. A. Memish, Remdesivir as a possible therapeutic option for the COVID-19, Travel. Med. Infect. Dis. 34 (2020) Article ID 101615; https://doi.org/10.1016/j. tmaid.2020.101615

33. T. Stanković, J. Dinić, A. Podolski-Renić, L. Musso, S. S. Burić, S. Dallavalle and M. Pešić, Dual inhibitors as a new challenge for cancer multidrug resistance treatment, Curr. Med. Chem. 26 (2019) 6074-6106; https://doi.org/10.2174/0929867325666180607094856

34. A. Chandwani and J. Shuter, Lopinavir/ritonavir in the treatment of HIV-1 infection: a review, Ther. Clin. Risk Manag. 4 (2008) 1023-1033; https://doi.org/10.2147/tcrm.s3285

35. J. Dorward and K. Gbinigie, Lopinavir/ritonavir: A rapid review of effectiveness in COVID-19 (on behalf of the Oxford COVID-19 Evidence Service Team Centre for Evidence-Based MedicineCEBM); https://www.cebm.net/covid-19/lopinavir-ritonavir-a-rapid-review-of-the-evidence-foreffectiveness-in-treating-covid/; last access date April 14, 2020

36. B. Cao, Y. Wang, D. Wen, W. Liu, J. Wang, G. Fan, L. Ruan, B Song, Y. Cai, M. Wei, X. Li, J. Xia, N. Chen, J. Xiang, T. Yu, T. Bai, X. Xie, L. Zhang, C. Li, Y. Yuan, H. Chen, H. Li, H. Huang, S. Tu, F. Gong, Y. Liu, Y. Wei, C. Dong, F. Zhou, X. Gu, J. Xu, Z. Liu, Y. Zhang, H. Li, L. Shang, K. Wang, K. Li, X. Zhou, X. Dong, Z. Qu, S. Lu, X. Hu, S. Ruan, S. Luo, J. Wu, L. Peng, F. Cheng, L. Pan, J. Zou, C. Jia, J. Wang, X. Liu, S. Wang, X. Wu, Q. Ge, J. He, H. Zhan, F. Qiu, L. Guo, C. Huang, T. Jaki, F. G. Hayden, P. W. Horby, D. Zhang and C. Wang, A trial of lopinavir-ritonavir in adults hospitalized with severe Covid-19, N. Engl. J. Med. 382 (2020) 1787-1799; https://doi.org/10.1056/NEJMoa2001282 